\title{
THE PRESENT RATE OF SUPERNOVAE
}

\author{
M. TURATTO ${ }^{1}$ \\ Osservatorio Astronomico di Padova \\ vicolo dell'Osservatorio 5, 35122 Padova, Italia
}

\begin{abstract}
We present and discuss the most recent determination of the rate of Supernovae in the local Universe. A comparison with other results shows a general agreement on the gross values but still significant differences on the values of the rates of various $\mathrm{SN}$ rates in different kinds of galaxies. The rate of SNe, used as a probe of Star Formation, confirms the young progenitor scenario for $\mathrm{SNII}+\mathrm{Ib} / \mathrm{c}$. The increasing diversity of SNe reflects also in the SN yields which may affect the chemical evolution of the Galaxy but, because of the limited statistics, we cannot estimate the contributions of the new subtypes yet. It is also expected that in a few years observational determinations of the $\mathrm{SN}$ rates at various look-back times will be available.
\end{abstract}

\section{Introduction}

In the context of the chemical evolution of the galaxies the supernova (SN) rate and its history play a fundamental role. But, while there are several theoretical models predicting the time evolution of the $\mathrm{SN}$ rates with time, observational determinations at different look-back times are not yet available. An estimate of the rate of SNIa at $\mathrm{z} \sim 0.4$ has only be published so far (Pain et al. 1997). The efforts of the high-z searches have been focused more on the detection and follow-up of the SNe for the determination of the geometry of the Universe than on the careful analysis of their databases for the SN rates.

Different is the case of the estimates of the rate in the local Universe. Searches of bright $\mathrm{SNe}$ are active since several decades and their archives have been exploited to this purpose since the sixties. The determinations of the rates have been carried out on different samples and with different techniques. In some case the differences among various estimates are larger 
than $100 \%$. In this paper we discuss the reasons of these uncertainties and their relation to the chemical evolution of the Galaxy.

\section{Definition of $\mathrm{SN}$ rate}

The SN rate is observationally defined in its general form as the number of $\mathrm{SNe}$ exploding in a given sample of galaxies during a certain time interval

$$
\nu\left(y r^{-1}\right)=N_{S N} N_{g a l}^{-1} \Delta t^{-1}
$$

and is measured in $\mathrm{yr}^{-1}$. However, since the galaxies can have very different stellar content, one expects that the average value depends strongly on the composition of the galaxy sample. Indeed, with the growing number of discoveries it appeared that critical parameters for the SN rates were the galaxy type, with late spirals more prolific than early spirals, and the galaxy luminosity, which in first approximation measures the number of available progenitor stars (Tammann 1970, Cappellaro \& Turatto 1988).

The SN rate can therefore be computed for each galaxy type as

$$
\nu^{\text {gtype }}(S N u)=N_{S N}^{g t y p e}\left(\sum_{j}^{N g a l} \sum_{i}^{\text {obs }} k_{i} L_{j}^{\text {gtype }} \Delta t_{i}^{\text {gtype }}\right)^{-1}
$$

where the index $j$ refers to the sample galaxies of that type and $i$ refers to the individual observations of each galaxy. Convenient units for the rate are the $\mathrm{SNu}$, where $1 \mathrm{SNu}$ corresponds to $1 \mathrm{SN}$ per $10^{10}$ solar luminosities per century. Because of the normalization to galaxy luminosity, $\mathrm{SNu}$ scale as $\mathrm{H}_{o}^{2}$. The parameter $k_{i}$ accounts for various observational selection effects.

\section{Efforts for better $\mathrm{SN}$ rates}

It is evident from the last equation that in order to have better estimates of the SN rates one can: 1) enlarge the statistical samples $\left(\mathrm{N}_{S N}, \mathrm{~N}_{\text {gal }}\right), 2$ ) estimate with higher precision the surveillance times, or 3) determine more reliable correction terms for the selection effects.

For the sample selection there are in practice two alternatives. We can, for instance, select all galaxies within a given volume, making reasonable assumptions on the surveillance times (cfr. Tammann 1994). Consequently the $\mathrm{SN}$ rate is computed using all the $\mathrm{SNe}$ discovered inside the volume during that period. The main advantage of this approach is in the large samples directly available form the galaxy and SN catalogues (e.g. Barbon et al. 1999). The drawback is in the critical assumption of the surveillance time which can hardly be the same for each galaxy. 
TABLE 1. The pooled SN searches

\begin{tabular}{llll}
\hline SN search & type $\left({ }^{*}\right)$ & SN discoveries $\left(^{* *}\right)$ & reference \\
\hline Asiago & pg & 51 & Cappellaro et al. 1993 A\&A 268, 472 \\
Crimea & pg & 33 & Tsvetkov 1983 SA 27, 22 \\
O.C.A. & pg & 16 & Pollas 1994, in Les Houches 1990, Bludman et al. p.769 \\
Calan/Tololo & pg & 12 & Hamuy et al. 1993 AJ 106, 2392 \\
Evans & vis & 54 & Evans 1997 PASA 14, 204 \\
\hline
\end{tabular}

$\left(^{*}\right) \mathrm{pg}=$ photographic; vis $=$ visual

$\left({ }^{* *}\right)$ some of which earlier reported by other searchers

The alternative is the survey approach which limits the galaxies to those surveyed by a specific SN search and the SNe to those discovered during the same search. The method allows for each galaxy a precise determination of the control time, i.e. the time during which a possible SN of a given type remains above the detection limit. This determination takes into account the distribution of the observations during the survey, the distance of the galaxy, the properties of the SN types and the observational limitations of the survey. The method, introduced already by Zwicky (1938), found several applications (e.g. Cappellaro \& Turatto 1988, Evans et al. 1989). In this case the drawback is the small size of the sample.

In order to overcome such limitation we developed a strategy to treat simultaneously the databases of several of SN searches (Cappellaro et al. 1993, 1997a). The use of the five SN searches listed in Tab. 1 led us (Cappellaro et al. 1999) to build the largest sample ever used for the determination of $\mathrm{SN}$ rates, $137 \mathrm{SNe}$ in $9346 \mathrm{RC} 3$ galaxies, for a total control time of the sample of $24954 \mathrm{yr}$.

The determination of the correction terms for the biases in the discovery of SNe is crucial and controversial. Two biases are by far the most important: the effect of inclination in spirals and the deficiency of discoveries in the central regions of distant galaxies.

Several authors claimed that the bias due inclination was not affecting visual and CCD searches but only photographic ones (Evans et al. 1989, Muller et al. 1992). The composite sample above allows us to perform a direct check handling separately photographic and the Evans' visual searches. The rate of SNe computed for the Evans' sample with the control-time technique described above is 2.6 times greater in face-on spirals than in inclined ones, showing that this bias is almost as severe as in photographic searches for which the factor is 2.9. The fact that different detectors provide similar results, is an indication that the effect is intrinsic to the galaxies rather 
than to the observational technique.

The natural explanation is that $\mathrm{SNe}$ in inclined spirals suffer in average heavier dust extinction than those in face-on galaxies, thus are more difficult to discover. If this interpretation is correct we expect (1) that searches working at longer wavelengths are less affected, (2) that the effect is stronger in late type spirals and (3) that it is more important for core-collapse $\mathrm{SNe}$ which are associated to a younger population, than for SNIa associated to an older population.

So far contradictory answers have been given to the points above. The effect seems stronger in late than in early type galaxies (Cappellaro et al. 1997a), only marginally smaller in the visual that in the blue bands, as said above, (probably significantly smaller for red CCD searches) but comparable for core-collapse and thermonuclear $\mathrm{SNe}$ (Cappellaro et al. 1997a). Larger statistics is necessary for unambiguous answers.

In the attempt to correct for the bias due to inclination we have tried two different approaches (Cappellaro et al. 1999). First we applied the sec $\alpha$ term corresponding to a plane parallel distribution of the dust in the disks of spirals. This results in an apparent overcorrection of the SN rate in edgeon galaxies. Then we explored the possibility to apply a model for the dust and the SN distributions consistent with our knowledge of the galaxies and SN progenitors. Even this more physical treatment showed some limitations indicating that the dust and the progenitor distributions in real galaxies are more complex than in simple models.

The study of the deficiency of SN discovery in the central regions of the parent galaxies has been discussed in detail in Cappellaro \& Turatto (1997). The effect is critical in distant galaxies with about $50 \%$ of SNe exploding in galaxies with recession velocities larger than $7500 \mathrm{~km} \mathrm{~s}^{-1}$ lost in the central parts, in particular, the innermost 10 arcsecs. Interestingly the effect seems correlated to the inclination in spirals with more inclined galaxies more affected. The effect for the SN searches of Tab.1 was empirically determined in Cappellaro et al. (1997a) and accounted for also in Cappellaro et al. (1999).

\section{The SN rates per unit B luminosity}

The SN rates derived with the procedure and the sample described above are reported in Table 2. The errors include those due to the statistics and those due to the input parameters and the selection effects.

A comparison of these rates with other recent determinations is shown in Figure 1. There is a reassuring overall agreement among rates based on different samples and with different recipes. The largest differences are with Tammann et al. (1994) and for SNIa in ellipticals and for SNII. We believe 
TABLE 2. SN rate(in $\mathrm{SNu}(\mathrm{B}))$ for the combined sample.

\begin{tabular}{|c|c|c|c|c|}
\hline galaxy & & rate $[\mathrm{SNu}]$ & & \\
\hline type & Ia & $\mathrm{Ib} / \mathrm{c}$ & II & All \\
\hline E-S0 & $0.18 \pm 0.06$ & $<0.01$ & $<0.02$ & $0.18 \pm 0.06$ \\
\hline $\mathrm{S} 0 \mathrm{a}-\mathrm{Sb}$ & $0.18 \pm 0.07$ & $0.11 \pm 0.06$ & $0.42 \pm 0.19$ & $0.72 \pm 0.21$ \\
\hline Sbc-Sd & $0.21 \pm 0.08$ & $0.14 \pm 0.07$ & $0.86 \pm 0.35$ & $1.21 \pm 0.37$ \\
\hline Sm,Irr,Pec & $0.40 \pm 0.16$ & $0.22 \pm 0.16$ & $0.65 \pm 0.39$ & $1.26 \pm 0.45$ \\
\hline
\end{tabular}

that such differences are mainly due to biases in the SN discoveries in the fiducial sample. Our new rates of SNIa show a shallower dependence on the galaxy types than our previous works of 1997 and 1993, which were based on the same approach but on smaller samples and with different bias corrections. At that time the increase of the rate of SNIa per unit luminosity moving from early to late type galaxies was considered as an argument in favor of different ages of the precursors of SNIa, with progenitors in spirals younger than those in ellipticals. This new result might be considered in favor of the conventional scenario of similarly old progenitors in all galaxy types. We warn that the high rate of SNIa observed in the heterogeneous class of Sm+Irregulars+Peculiars is uncertain to $\pm 40 \%$.

As in the previous works, the rates of SNII increase from early to late spirals but now the peak values are smaller. This is mainly due to the smaller correction terms used for the inclination effect. Similar trends are shown also by SNIb/c which however remain definitely less frequent than type Ia.

\section{SN rates vs. Star Formation Rate}

The normalization of the rates to the B luminosity of the galaxies has been a natural choice. In fact, the B magnitude is the magnitude most frequently available in the literature and it scales, in first approximation, with the galaxy mass since stars of different population have significant emission in this band.

In principle, the normalization of the rates to photometric bands sensitive to specific stellar populations might give important insights onto the SN progenitor scenario. If in all galaxies the flux at a given wavelength were due to a single stellar population, then the rate of the SNe associated to this population, normalized to the luminosity in this band, should be constant. For instance, infrared $\mathrm{H}$ or $\mathrm{K}$ bands would be particularly suit- 


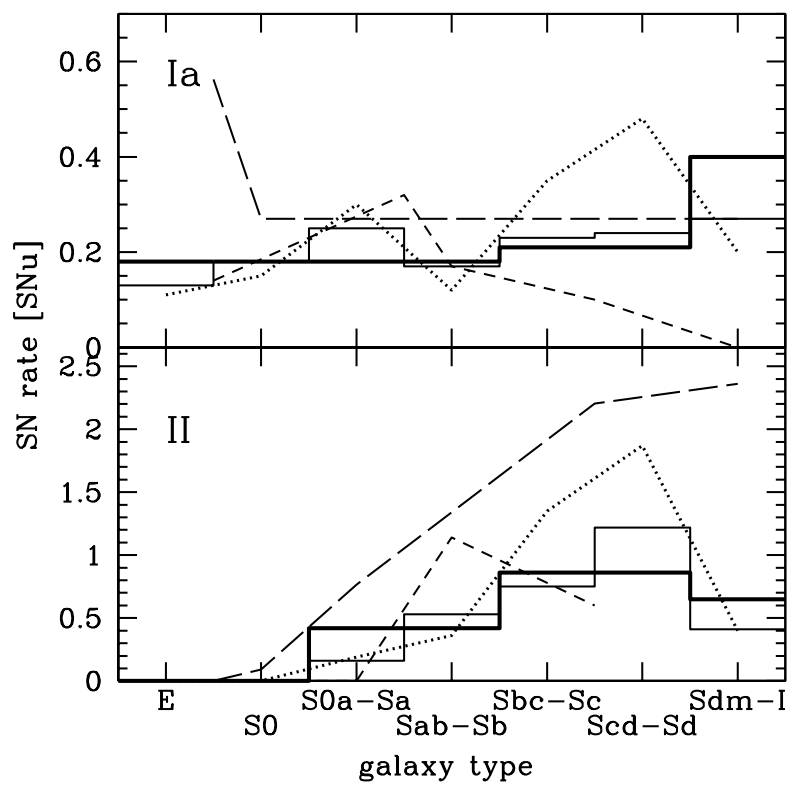

Figure 1. Comparison between different recent estimates of the SN rates scaled to $\mathrm{H}-o=75 \mathrm{~km} \mathrm{~s}^{-1} \mathrm{Mpc}^{-1}$ (Cappellaro et al. 1999, thick continuos line; Cappellaro et al. 1997a, thin continuous line; Tammann et al. 1994, long dashed line; van den Bergh \& McClure 1994, short dashed line; Cappellaro et al. 1993, dotted line).

able for the study of the type Ia progenitors since they sample well the old stellar population. Unfortunately, the sample of galaxies with published infrared photometry is still too small for any direct analysis. Different is the situation for the integrated far infrared luminosities which are available for a large sample of galaxies thanks to the IRAS survey. $\mathrm{L}_{F I R}$ has been used often as an indicator of SFR and therefore we may try to gain information on the progenitors of core-collapse SNe.

Contrary to the expectations, the rate of core-collapse SNe (cfr. column 3 of Table 3) varies of almost a factor two moving from early and late spirals. Taken to face value this means that SNII and Ib/c are not associated to massive progenitors. Actually we think that this inconsistency is due to the contamination of the FIR emission associated with dust around massive stars by the cool component associated to the general stellar radiation field. In other words we consider this a further evidence that in normal galaxies $\mathrm{L}_{F I R}$ is not a reliable probe of $\mathrm{SFR}$. 
TABLE 3. SN rates per unit FIR luminosity.

\begin{tabular}{|c|c|c|c|}
\hline \multirow{2}{*}{$\begin{array}{l}\text { galaxy } \\
\text { type }\end{array}$} & \multicolumn{3}{|c|}{ SN rate $[\mathrm{SNuIR}]$} \\
\hline & $\mathrm{Ia}$ & $\mathrm{II}+\mathrm{Ib} / \mathrm{c}$ & All \\
\hline E-S0 & $1.8 \pm 0.8$ & & $1.8 \pm 0.8$ \\
\hline $\mathrm{SOa}-\mathrm{Sb}$ & $0.6 \pm 0.2$ & $2.0 \pm 0.5$ & $2.7 \pm 0.5$ \\
\hline Sbc-Sd & $0.6 \pm 0.1$ & $3.5 \pm 0.6$ & $4.1 \pm 0.6$ \\
\hline
\end{tabular}

This finding is supported by the following argument. The effect of the general radiation field, due also to old stars, can be partially removed normalizing the FIR to the B luminosity to get the so-called infrared excess. The $\mathrm{SN}$ rates (in $\mathrm{SNu}(\mathrm{B})$ ) computed independently for the three classes of IRAS not detected, low and high infrared excess galaxies are similar for type Ia. Instead for type II+Ib/c SNe the rate of IRAS not detected galaxies is significantly lower than in galaxies with high FIR excess (Table 6 of Cappellaro et al. 1999) in agreement with the young progenitor scenario.

Also the colors of galaxies can be used as indicators of the stellar populations with bluer galaxies hosting younger, more massive stars. We computed the $\mathrm{SN}$ rates (in $\mathrm{SNu}(\mathrm{B})$ ) in galaxies with different optical integrated colors (Cappellaro et al. 1999). We found that the observed rate of SNIa is independent on the galaxy colors while the rate of core-collapse $\mathrm{SNe}$ is higher in bluer spirals than in galaxies of the same morphological type but with redder colors.

It is also interesting to note that the number of massive progenitors predicted by the star formation rate, derived by evolutionary synthesis models of galaxies with different colors, closely matches the observed number of core-collapse SNe.

\section{Frequency and Nucleosynthesis}

Supernovae are primary contributors to the chemical enrichment of the galaxies because they return to the ISM the elements synthesized by the hydrostatic and explosive burning processes, with the exception of the material trapped in the condensed remnants of core-collapse SNe. From a general point of view core-collapse SNe, descending from young massive progenitors determine the enrichment in the early galactic evolution while supernovae of type Ia are responsible of the heavy elements enrichment in the later phases of the galactic evolution on time scales which depend on the binary configuration and mass ratios. Current efforts are devoted to 
determine the masses of enriched material released by different kinds of explosions given their relative frequency.

For type II it is expected that the yields vary with the stellar mass, mass cut, explosion energy and neutron excess (Nakamura et al. 1999). The effect of these parameters have been modeled theoretically but observational confirmations are still needed. The well studied explosions of SN 1987A, SN 1993J and SN 1994I with progenitor masses between 13 and $20 M_{\odot}$ produced about $0.07 M_{\odot}$ of ${ }^{56} \mathrm{Ni}$. Early works on the late light curves indicated that most SNII produced similar amounts of radioactive material (Turatto et al. 1990). In the last years data have been accumulated which show a different scenario (Danziger, this meeting). In particular there seems to be an increase of the ${ }^{56} \mathrm{Ni}$ production for stars up to about $25 M_{\odot}$ (e.g. SN 1997ef, $0.15 M_{\odot}$ of ${ }^{56} \mathrm{Ni}$, and $\left.1998 \mathrm{bw}, 0.7 M_{\odot}\right)$ after which the gravitational potential wins and a small ${ }^{56} \mathrm{Ni}$ mass is ejected due to fallback (e.g. SN 1997D, 0.002 $M_{\odot}$ of ${ }^{56} \mathrm{Ni}$; Turatto et al. 1998). The variation of the mass cut as a function of the progenitor mass seems to account for the observed trends of iron peak element abundance ratios in stars of low metallicity (Nakamura et al. 1999). The study of a second object with an expected low ${ }^{56} \mathrm{Ni}$ production, SN 1999eu a twin of SN 1997D at early time, will help to strengthen this hypothesis. Unfortunately the objects for which both the progenitor mass and the ejected ${ }^{56} \mathrm{Ni}$ mass are available is so limited that no kind of statistics is still possible.

Also for type Ia SNe, once considered a very homogeneous class, there is now evidence that the yields can be different. Cappellaro et al. (1997b) showed that the photometric properties of SNIa require a range of a factor 10 of the radioactive material synthesized during the explosion ( 0.1 to 1 $\left.M_{\odot}\right)$ and a factor 2 in the total mass of the ejecta. From a theoretical point of view such variations can be accounted for with different precursor masses (Chandrasekhar and sub-Chandrasekhar), and flame speeds. An attempt to determine the relative frequency of different Type Ia subtypes has been performed by Cappellaro et al. (1997a). Again going to analyze very particular SN subtypes we faced the problem of poor statistics. For faint SNIa, producing small amounts of ${ }^{56} \mathrm{Ni}$, we found an intrinsic rate about $1 / 4$ of that of all SNIa although they constitute only $5 \%$ of all SNIa discoveries. Also the percentage of SNIa producing large amounts of ${ }^{56} \mathrm{Ni}$ is expected to be small. In fact the observed number is relatively small although they are brighter than the average, hence easier to discover.

\section{Conclusions}

The determinations by various authors of the rates of SNe in the local Universe are in substantial agreement although large differences are still present 
on the rates of individual SN types in specific galaxy types. Improvements are possible in the treatment of the databases and in the analyses of the selection effects. However the slow increase of the statistics at low redshifts is the limiting factor.

On the contrary, huge is the number of discoveries at high $(\mathrm{z}=0.5-$ 1.0) redshifts thanks to the projects aiming to determine the geometry of the Universe with the use of $\mathrm{SNe}$ as distance indicators. The analysis of these databases can already allow the determination of the rates at such early epochs. Data at intermediate redshifts are still missing. In order to cover this gap we have started a SN search in the southern hemisphere which is starting to produce the first interesting results (SNe 1999ey, 1999gt, 1999gu). In few years observational estimates of the SN rates at various look-back times will provide strong constraints to the Galaxy evolutionary models.

\section{References}

1. Barbon,R., Buondí, V., Cappellaro, E., Turatto, M., 1999, A\& AS 139, 531

2. Cappellaro, E., Turatto, M., 1988, A\&A 190, 10

3. Cappellaro, E., Turatto, M. 1997, in: Nato - ASI on Thermonuclear Supernovae, eds. R. Canal, P. Ruiz-Lapuente, J. Isern, Kluwer Academic Publisher, Dordrecht p. 77

4. Cappellaro, E., Turatto, Benetti,S., Tsvetkov, D.Yu., Bartunov, O.S., Makarova, I.N., 1993, A\&A 273, 383

5. Cappellaro, E., Turatto, M., Tsvetkov, D.Yu., Bartunov, O.S., Pollas, C., Evans, R., Hamuy, M., 1997a, A\&A 322, 431

6. Cappellaro, E., Mazzali,P., Benetti,S., Danziger, I.J., Turatto, M., Della Valle,M., Patat,F., 1997b, A\&A 328, 203

7. Cappellaro, E., Evans,R., Turatto, M., 1999, A\&A 351, 459

8. Evans,R., van den Bergh,S., McClure,R.D., 1989, ApJ 345, 752

9. Muller, R.A., Marvin, H.J., Pennypacker, C.R., Perlmutter, S., Sasseen, T.P., Smith, C.K. 1992, ApJ 384, L9

10. Nakamura,T., Umeda,H., Nomoto,K., Thielemann,F., Burrows,A., 1999, ApJ 517, 193

11. Pain, R., et al. 1996, ApJ, 473, 356

12. Tammann, G.A., 1970, A\&A 8, 458

13. Tammann, G.A., Löfller,W., Schröder,A., 1994, ApJS 92, 487

14. Turatto,M., Cappellaro,E., Barbon,R., Della Valle,M., Ortolani,S., Rosino,L., 1990, AJ 100, 771

15. Turatto,M., et al. 1998, ApJL 498, 129

16. van den Bergh, S., McClure, R.D. 1994, ApJ 425, 205

17. Zwicky,F., 1938, ApJ 96, 28 